\title{
Confusion: delirium and dementia - a smartphone app to improve cognitive assessment
}

\author{
Selina Sangha, J George, Craig Winthrop, Sonia Panchal
}

North Cumbria University Hospitals Trust

\begin{abstract}
Older patients with dementia and delirium are more prone to adverse events in hospital, but formal cognitive assessment to identify these vulnerable patients on admission is often not carried out by junior doctors. A smartphone app was created and provided on hospital wards to facilitate the use of standard cognitive assessments for delirium and dementia. Before the introduction of the app, $36 \%$ of patients over 75 years old were assessed cognitively. After the app, the percentage of cognitive assessments improved to $63 \%$. Improvements in cognitive assessments were most marked after individual teaching of the doctors on the wards in the use of the app and on making the app available on the ward tablets. The results of the study suggest that the introduction of a smartphone app for junior doctors can improve performance in cognitive assessment of older people.
\end{abstract}

\section{Problem}

Cognitive assessment is very important in older people admitted to hospital particularly to detect dementia and delirium and should be a routine part of their care.[1] Unfortunately, national audits have suggested that a formal cognitive assessment is only carried out in around $50 \%$ of patients at risk.[2]

\section{Background}

Early diagnosis of dementia is emphasised in the National Dementia Strategy and is a cornerstone of dementia care and safeguarding patients.[3] Patients with dementia are more prone to adverse events in hospital [4] and they may have difficulty in understanding their treatment and granting consent. These problems may be overlooked if cognitive impairment is not routinely tested. Older patients, especially those with dementia, are more prone to develop delirium (acute confusional state), which may be due to underlying illness and may be missed.[5] Patients with dementia and delirium are also prone to develop complications, such as hospital acquired infections which can increase mortality and length of stay.[4] For these reasons, older people should be routinely screened for delirium and dementia, particularly in acute elderly and orthopaedic trauma wards where these disorders are common.

\section{Baseline measurement}

Two entirely different wards were selected for the project. The first is a specialised elderly care ward that regularly deals with patients with dementia and delirium, and where the staff are relatively familiar with cognitive assessment. The second ward is a trauma ward mainly for elderly people with femoral neck fractures where the junior doctors are more surgically orientated and less familiar with cognitive assessment. All notes for patients over 75 years were independently assessed by a coding clerk who recorded over a four month period the percentage of patients over 75 receiving a cognitive assessment including an assessment for delirium using the confusion assessment method (CAM). Full cognitive assessments, including screening for delirium, were carried out before the intervention on 26 out of 71 patients over 75 years (36\%)

\section{Design}

In order to make cognitive assessment easier and more applicable, a smartphone app was designed that facilitated the use of the standard assessment instruments for delirium. It is based on the NICE guidelines on delirium and includes the confusion assessment method (CAM) which is recommended for delirium assessment [5], along with the six item screener which is commonly used for cognitive assessment in emergency care.[6] The app also includes management advice for patients with dementia and delirium, as well as a simple formulary of drugs to avoid that can worsen confusion. In addition, the app incorporates an example teaching case history which when completed allows the user to download a certificate for their education portfolio. The "Confusion" app is free and can be used on smartphones and tablets. It is available for Apple and Android devices and can be found on their respective app stores. The project was supported by Health Education England who awarded funding through the "Better Training Better Care, Inspire Improvement!" project.

\section{Strategy}

PDSA cycle 1: Initial education and awareness talks of the importance of cognitive assessment in acute older patients admitted to hospital were given to the foundation doctors.

PDSA cycle 2: In this cycle, the education given to the doctors was much more specific and given to individual doctors working in orthopaedics and elderly care. This included not only the 
importance of cognitive assessment looking for delirium and dementia and why we needed to do this, but also the use of the app and the particular cognitive instruments available on the app. Also, the use of the app to download a certificate of learning was demonstrated to the doctors.

PDSA cycle 3: We realised that although the majority of doctors used smartphones, some did not, so the app was downloaded on the ward tablets to improve access to the cognitive assessment instruments.

The junior doctors in the hospital were also sent a questionnaire at the end of the study on whether they found the app useful for cognitive assessments, and how often they used it.

\section{Post-measurement}

The number of cognitive assessments complete and clearly recorded in the notes was:

Month 1 - [Baseline] Elderly care ward: 66\% (8/12), Trauma ward: $30 \%$ (18/59), Total: $36 \%(26 / 71)$

Month 2 - [PDSA 1] Elderly care ward: 50\% (8/16), Trauma ward: $28 \%$ (12/42), Total: $34 \%(20 / 58)$

Month 3 - [PDSA 2] Elderly care ward: 73\% (11/15), Trauma ward: $51 \%(30 / 59)$, Total: $55 \%(41 / 74)$

Month 4 - [PDSA 3] Elderly care ward: 95\% (19/20), Trauma ward: $53 \%$ (34/64), Total: $63 \%(53 / 84)$

The percentage of patients fully cognitively assessed on both wards increased from $36 \%$ (month 1) to $63 \%$ (month 4).

Questionnaires were sent to 35 doctors working in medicine, elderly care, and surgery. Seventeen doctors (49\%) responded to the questionnaire and had all used the app. Eleven of the doctors had used the app more than five times, and three more than 10 times. All the doctors found the app easy to use to help with admission clerking and 16 out of 17 found the illustrative case scenario useful. Ten doctors downloaded the case study certificate of completion for their portfolio and all the doctors described their knowledge improved following use of the app.

See supplementary file: ds4667.pdf - "Cognitive assessment of patients over 4 months and Confusion app screenshots"

\section{Lessons and limitations}

The smartphone app was designed to improve patient care by encouraging cognitive assessment of older people particularly for delirium and dementia. This aim was at least partially achieved in that cognitive assessment by junior doctors improved on both the specialist elderly ward and on the orthopaedic trauma ward. Initial results were disappointing (PDSA cycle 1) and it was not until the doctors were introduced to the app with individual teaching that cognitive assessment rates improved (PDSA cycle 2). They improved further when the app was made available on the ward tablets and owning a smartphone was not necessary (PDSA cycle 3). Although we did not achieve 100\%, cognitive assessments did improve considerably.

On the specialist elderly care ward $95 \%$ was achieved and on the orthopaedic ward $53 \%$ was achieved after four months. There may be a number of reasons for this as with very ill patients it is not always possible for them to cooperate fully and also in some cases the results may not have been filed in the notes. Nevertheless there was a considerable improvement in both wards especially when the app was downloaded on the ward tablets. Improvement in cognitive assessment was not as marked on the trauma ward compared to the elderly care ward and this possibly was because the patients were clerked by many different doctors on this ward, and often just before the patients were going to theatre, when time was at a premium.

The response rate to the questionnaire (49\%) was disappointing. This may have been because not all the doctors had smartphones, although tablet versions were available on the orthopaedic and geriatric wards. Even so, among the doctors that replied to the questionnaire, all found the app very easy to use and increased their knowledge.

It is not possible to be absolutely certain that it was the use of the app which resulted in the increased cognitive scoring, although the positive responses from the questionnaire suggests it at least played a part.

\section{Conclusion}

The results of this study suggest that the introduction and use of a smartphone app for junior doctors increases the likelihood of older patients receiving a cognitive assessment for detecting delirium and dementia on specialist elderly and orthopaedic trauma wards. We intend to promote use of the app by nurses and therapists as well as doctors, and encourage wider use of this app in other hospitals.

\section{References}

1. Urgent Care Pathways for Older People with complex needs. Best Practice Guidance. Department of Health 2007.

2. National Audit of Dementia Care in General Hospitals 2012-2013. Second Round Audit Report. Royal College of Psychiatrists 2013.

3. Living well with dementia: A national dementia strategy. Department of Health 2009

4. George J, Long S, Vincent C. How can we keep patients with dementia safe in our acute hospitals? A review of challenges and solutions. JRSM 2013;106(9):355-61.

5. NICE Guidelines on Delirium. July 2010.

6. Wilber ST, Carpenter CR, Hastey FM. The Six Item Screener to detect cognitive impairment in older adults in an emergency department. Ann Emerg Med 2008;15:613-6. 


\section{Declaration of interests}

Nothing to declare.

\section{Acknowledgements}

Health Education England for providing funding as part of the

'Better Training, Better Care, Inspire Improvement!' programme 